\title{
Reduced oligodendroglial density in the inferior parietal lobule and lack of insight in schizophrenia
}

\author{
Victor M. Vostrikov, MD, PhD \\ Natalya S. Kolomeets, MD, PhD \\ Natalya A. Uranova MD, PhD \\ Laboratory of Clinical Neuropathology, \\ Mental Health Research Center, Moscow \\ RUSSIA
}

\begin{abstract}
Background and Objectives: Alterations and deficits of oligodendrocytes reported in the grey and white matter in schizophrenia may contribute to neuronal disconnectivity. Prefrontal-parietal functional disconnections have been implicated in diverse clinical symptoms of schizophrenia, including poor insight. We studied the effects of schizophrenia diagnosis and insight on numerical density $(\mathrm{Nv})$ of oligodendrocytes in the inferior parietal lobule (IPL).

Methods: Nissl-stained sections from the Stanley "Parietal Collection" from male schizophrenia subjects $(n=24)$ having poor, fair, or good insight and healthy matched controls $(n=24)$ were examined. The Nv of oligodendrocytes was estimated in layer 3 of BA 39 and BA 40 of the IPL and in white matter underlying layer 6 by optical dissector method.

Results: In BA 39 we found a significant $15 \%$ decrease in the Nv of oligodendrocytes in layer 3 in the schizophrenia group. Nv of oligodendrocytes in the poor+fair insight subgroup was $20 \%$ lower compared to controls $(\mathrm{p}<0.05)$ and to good insight subgroup $(\mathrm{p}=$ $0.055)$. Nv of oligodendrocytes in the good insight subgroup did not differ from the control group. A significant lateralization of oligodendrocyte density was detected in layer 3 $(\mathrm{L}>\mathrm{R})$ only in the control group. There were no significant group differences in the Nv of oligodendrocytes in BA 40 or in the white matter underlying BA 39/40 areas.

Conclusions: Lack of insight in schizophrenia may be associated with a deficit of oligodendroglia in the grey matter of IPL.
\end{abstract}

Received: 20 February 2012

Revised: 14 July 2012

Accepted: 18 July 2012

This work was supported by the Stanley Medical Research Institute. Grant number: 07R-1787. 


\section{Background}

Schizophrenia is believed to be associated with altered neuronal connectivity. Some research suggests that several neural activity deficits found in schizophrenia may be associated with prefrontal and inferior parietal lobe dysfunction ${ }^{1}$. The inferior parietal lobule (IPL) and the prefrontal cortex (PFC) have extensive interconnections and common cortical and subcortical target regions ${ }^{2}$. The IPL consists of the supramarginal gyrus (BA 40) and adjacent angular gyrus (BA 39), and it participates in sensory integration, body image, concept of self and in executive functions (see Torrey 2007 for review) ${ }^{3}$. The IPL is among the most highly lateralized areas of the brain, and both decreased cerebral lateralization and reversed asymmetry of the IPL have been reported in schizophrenia ${ }^{4}$.

Imaging studies demonstrated reduced white matter integrity in the IPL in schizophrenia patients ${ }^{5}$ a significantly greater decreases over time in parietal white matter ${ }^{6}$ and no changes in white matter volume of parietal regions in schizophrenia subjects ${ }^{4}$.

At least 14 studies have reported anatomical deficits associated with lack of insight in individuals with schizophrenia. A study using computerized tomography (CT) reported frontal lobe atrophy ${ }^{7}$. Eleven studies used magnetic resonance imaging (MRI); three reported that lack of insight was associated with ventricular enlargement ${ }^{8}$, brain size ${ }^{9}$ or cortical thickness ${ }^{10}$, four reported associations with frontal lobe structures ${ }^{11-14}$, and the cingulate and precuneus ${ }^{15-17}$ and/or temporal and inferior parietal areas ${ }^{18}$. One study used functional magnetic resonance imaging (fMRI) to demonstrate improved functioning of the medial prefrontal cortex during tasks assessing awareness of illness in individuals with schizophrenia acutely ill and then clinically improved ${ }^{19}$. Another study used diffusion tensor imaging (DTI) to demonstrate an association between widespread white matter dysfunction and decreased awareness of illness ${ }^{20}$. Finally, a recent study used single photon emission computed tomography (SPECT) to demonstrate an association between decreased awareness of illness and decreased blood flow in the precuneus ${ }^{21}$. The present study is the first to use neuropathological methods to assess awareness of illness in individuals with schizophrenia.

Postmortem and genetic studies have reported alterations and deficits of oligodendrocytes in the pathophysiology of schizophrenia $^{22-26}$. However, the association of oligodendrocyte abnormalities with clinical symptoms of schizophrenia, particularly poor insight, remains uncertain. Poor insight is associated with more severe negative symptoms in patients with schizophrenia, poorer prefrontal cognitive functioning, lower prefrontal volumes ${ }^{27}$, reduced gray matter volume in the parietal regions in schizophrenia patients $^{4,18}$. Consistent with these data is a deficit of pericapillary oligodendrocytes previously reported in the PFC in the subgroup of schizophrenia subjects with predominantly negative symptoms compared to controls ${ }^{26}$. Previously we reported a reduction in the number of perineuronal oligodendrocytes in layer 3 of the dorsolateral PFC in schizophrenia ${ }^{25}$. Based on these data, we hypothesized that a deficit of oligodendrocytes might occur in the IPL in schizophrenia similar to that in the PFC and that this deficit might be more pronounced in schizophrenia subjects having poor insight than in subjects having good insight.

We studied the effects of schizophrenia diagnosis, insight, and hemisphere on oligodendrocyte density in BA 39 and BA 40 in layer 3 and in the white matter underlying layer 6 . 


\section{Materials and methods}

\section{Samples}

Human brain specimens were donated by the Stanley Medical Research Institute's "Parietal Collection". The samples consisted of 48 subjects ( 24 controls and 24 with schizophrenia). Diagnosis was made according to DSM-IV criteria. A postmortem assessment of each person's awareness of illness (insight) was done by E. Fuller Torrey MD, a senior psychiatrist who did a diagnostic summary of each brain in the Stanley Brain Collection. Medical records and, in approximately half of all cases, interviews with family members, were utilized to make diagnoses. In the course of doing the diagnostic assessment indications of the person's awareness of illness were noted. These included such things as the person's explicit acceptance or denial of illness; acceptance of denial of the need for medication; refusal to take medication; and failure to follow treatment plans, often leading to mulitiple re-admissions. After all available information had been considered the person's awareness of illness was categorized as either good (definite evidence of acceptance of illness); poor (definite evidence suggesting non acceptance of illness); fair (suggestions of non acceptance of illness but less conclusive and/or more ambiguous); or unknown (information insufficient to make a judgment). All diagnoses and assessments of awareness of illness were finalized before any neuropathological studies were done on the brains. The demographic and clinical data are given in Table 1.

The brain specimens were coded, and all cytoarchitectural assessments were done blindly. Tissue was available from one hemisphere of each brain. The angular gyrus (BA 39 ) and supramarginal gyrus (BA 40) were identified according to macroscopic land- marks ${ }^{28}$. Ten serial sections through the IPL (each 17 th section) were mounted on slides and Nissl-stained.

\section{Stereological analysis}

The sublayers of layer 3 ( $a, b$ and $c$ ) in BA 39 and BA 40 were easily identified. The numerical density $(\mathrm{Nv})$ of oligodendrocytes was estimated in BA 39 and BA 40 in each sublayer of layer 3 and in the white matter underlying BA 39/40 using an optical disector method. The sections were viewed on a Carl Zeiss Axio Imager M1 microscope with a computer-guided microscopy system AxioVision. Prior to the actual analysis, the optimal parameters for counting box size were determined. Section thickness was measured on slides as ranging from 14-16 $\mu \mathrm{m}$. Grid sizes were $55 \times 55 \mu \mathrm{m}$, and dissector of depth $10 \mu \mathrm{m}$, guard strata above and below the dissector averaged at $4 \mu \mathrm{m}$. Sections were examined under $100 \times 1.4$ oil immersion objective. The identification of oligodendrocytes and optical dissector method have been described previously ${ }^{29} .100$ fields were counted per each sublayer of layer 3 and 100 fields per white matter underlying layer 6 per case.

\section{Statistical analyses}

Statistical analysis was performed using Statistica (Version 7). The data were examined using the Kolmogorov-Smirnov test for normality. A Pearson or Spearman correlation analyses were performed to assess correlations between the parameter measured and age, postmortem interval, $\mathrm{pH}$, refrigerator interval, brain weight, lifetime antipsychotics, age at onset and duration of disease. Comparisons between patients with schizophrenia and controls were performed using MANOVA with Nv of oligodendrocytes in 
Table 1

Demographic and clinical information on the schizophrenia and control cases

Group

\begin{tabular}{|c|c|c|c|}
\hline & & Controls $(\mathrm{n}=24)$ & Schizophrenia $(n=24)$ \\
\hline \multirow[t]{7}{*}{ Demographic } & Age (yrs) & $44.3 \pm 9.3$ & $39.8 \pm 10.7$ \\
\hline & Hemisphere side & $10 \mathrm{R} / 14 \mathrm{~L}$ & $10 \mathrm{R} / 14 \mathrm{~L}$ \\
\hline & PMI (hours) & $24.4 \pm 10.8$ & $29.1 \pm 11.6$ \\
\hline & Fixation time (months) & $\begin{array}{l}64.7(22-90) \text { left } \\
50.4(21-83) \text { right }\end{array}$ & $\begin{array}{l}60.1(19-91) \text { left } \\
51.3(24-88) \text { right }\end{array}$ \\
\hline & Refrigerator interval (hours) & $6.1 \pm 4.9(18-1)$ & $6.9 \pm 4.8(18-1)$ \\
\hline & Brain weight $(\mathrm{g})$ & $1479.3 \pm 102.9$ & $1469.5 \pm 100.8$ \\
\hline & Brain $\mathrm{pH}$ & $6.67 \pm 0.2$ & $6.52 \pm 0.2$ \\
\hline \multirow[t]{8}{*}{ Clinical } & Cause of death & $a / 21, b / 2, c / 0, d / 1$ & $a / 13, b / 0, c / 6, d / 5$ \\
\hline & Duration of illness (yrs) & $0 \pm 0$ & $\begin{array}{l}20.8 \pm 10.3 \\
(35-1)\end{array}$ \\
\hline & Age of onset (yrs) & $0 \pm 0$ & $\begin{array}{l}19.0 \pm 5.8 \\
(34-9)\end{array}$ \\
\hline & Time in hospital (yrs) & $0 \pm 0$ & $\begin{array}{l}1.3 \pm 2.7 \\
(12-0)\end{array}$ \\
\hline & Lifetime antipsychotics & $0 \pm 0$ & $\begin{array}{l}79002 \pm 79529 \\
(50-300000)\end{array}$ \\
\hline & Lifetime alcohol use & $\begin{array}{l}0 / 15,1 / 3,2 / 0,3 / 2, \\
4 / 3,5 / 1\end{array}$ & $\begin{array}{l}0 / 6,1 / 2,2 / 3,3 / 4, \\
4 / 3,5 / 6\end{array}$ \\
\hline & Lifetime drug use & $\begin{array}{l}0 / 18,1 / 2,2 / 1,3 / 3, \\
4 / 0,5 / 0\end{array}$ & $\begin{array}{l}0 / 6,1 / 3,2 / 3,3 / 6 \\
4 / 3,5 / 3\end{array}$ \\
\hline & Smoking at time of death & $0 / 12,1 / 5,9 / 7$ & $0 / 2,1 / 18,9 / 4$ \\
\hline
\end{tabular}

Antipsychotic dose is lifetime dose in fluphenazine equivalent (mg), lifetime alcohol use and lifetime drug use: 0 - little or none, 1 - social, 2 - moderate past, 3 - moderate present, 4 - heavy past, 5 - heavy present; smoking at time of death: 0 - no, 1 - yes, 9 - unknown. Cause of death is categorized under the following headings: a - cardio-pulmonary, b - accident, $\mathrm{c}$ - suicide, $\mathrm{d}$ - other.

three sublayers of layer 3 as the dependent variables, and diagnosis and hemispheres as the independent variables. A one-way ANOVA followed by post hoc Duncan's test was used for white matter, to compare the control group and three insight schizophrenia subgroups (poor, fair and good insight).

\section{Results}

\section{Effects of disease and hemispheres}

The mean Nv of oligodendrocytes and group comparisons for sublayers of layer 3 and for the white matter underlying layer 6 are given in Table 2. A significant effect of diagnosis on the $\mathrm{Nv}$ of oligodendrocytes in sublayers of layer 
Table 2

Effect of diagnosis on the $\mathrm{Nv}$ of oligodendrocytes

\begin{tabular}{lcccc} 
Layers & Controls \pm SD $(\mathrm{n}=24)$ & Schizophrenia $\pm \mathrm{SD}(\mathrm{n}=24)$ & $\mathrm{F}(1,44)$ & $\mathrm{p}$ \\
\hline 3a & Brodmann's area 39 & & \\
\hline $\mathrm{b}$ & $126.95 \pm 28.0$ & $109.83 \pm 32.3$ & 4.24 & $\mathbf{0 . 0 4 5}$ \\
$3 \mathrm{c}$ & $139.66 \pm 33.4$ & $118.59 \pm 34.6$ & 4.79 & $\mathbf{0 . 0 3 4}$ \\
\hline White matter & $152.01 \pm 35.1$ & $130.2 \pm 42.8$ & 4.11 & $\mathbf{0 . 0 4 9}$ \\
\hline & $412.64 \pm 69.3$ & $392.6 \pm 47.7$ & 1.36 & 0.24 \\
\hline 3a & $154.89 \pm 29.7$ & Brodmann's area 40 & & \\
3b & $148,78 \pm 25.15$ & 0.63 & 0.43 \\
3c & $161.96 \pm 29.0$ & $158.21 \pm 24.1$ & 0.22 & 0.64 \\
\hline White matter & $386.75 \pm 54.1$ & $183.99 \pm 26.2$ & 0.36 & 0.55 \\
\hline & & $398.13 \pm 43.5$ & 0.5 & 0.48 \\
\hline
\end{tabular}

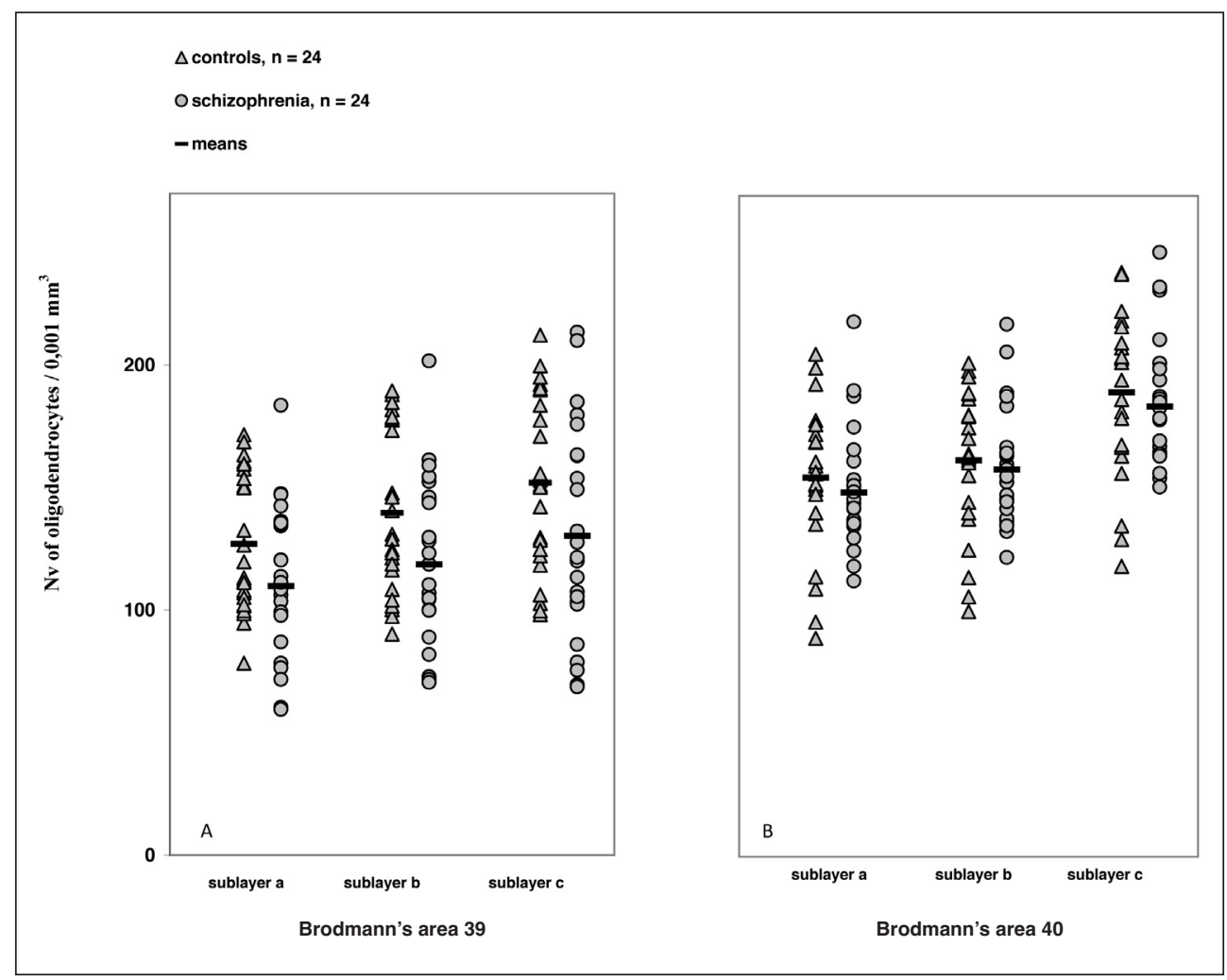

Figure 1. Plots of individual values and means (lines) of the Nv of oligodendrocytes in three sublayers of layer 3 in BA 39 (A) and in BA 40 (B). 
3 was found in BA 39 but not in BA 40 . There was a significant $15 \%$ decrease of the Nv of oligodendrocytes in each sublayer of BA 39 in the schizophrenia group compared to controls. The Nv oligodendrocytes did not differ significantly between the groups in the white matter underlying layer 6 of BA 39 and BA 40 (Table 2, Figure 1).

There was a significant hemisphere effect on the Nv of oligodendrocytes in layer 3
( $\mathrm{F} \geq 7.95, \mathrm{p}<0.01$ for each sublayer). A post hoc test demonstrated a significant hemispheric difference in the control group, but not in the schizophrenia group: the $\mathrm{Nv}$ of the oligodendrocytes was significantly higher in the left hemisphere compared to the right hemisphere in $3 b$ and $3 c(22 \%, p<0.03)$ and nonsignificantly higher in $3 \mathrm{a}(17 \%, \mathrm{p}<0.07)$. There were no significant hemispheric differences in BA 40 (Table 3).

Table 3

Comparison of the Nv of oligodendrocytes between left and right hemispheres in BA 39

\begin{tabular}{lllllll} 
Sublayers & Control group & \multicolumn{5}{l}{ Schizophrenia group } \\
\hline $\begin{array}{l}\text { Left } \\
\text { hemisphere } \\
(\mathrm{n}=14)\end{array}$ & $\begin{array}{l}\text { Right } \\
\text { hemisphere } \\
(\mathrm{n}=10)\end{array}$ & $\mathrm{p}$ & $\begin{array}{l}\text { Left } \\
\text { hemisphere } \\
(\mathrm{n}=14)\end{array}$ & $\begin{array}{l}\text { Right } \\
\text { hemisphere } \\
(\mathrm{n}=10)\end{array}$ & $\mathrm{p}$ \\
\hline $3 \mathrm{a}$ & $136.6 \pm 28.5$ & $113.4 \pm 22.2$ & 0.07 & $119.8 \pm 36.4$ & $95.9 \pm 19.6$ & 0.06 \\
\hline $3 \mathrm{~b}$ & $153.3 \pm 31.3$ & $120.5 \pm 27.2$ & $\mathbf{0 . 0 2}$ & $128.1 \pm 41.1$ & $105.3 \pm 16.7$ & 0.1 \\
\hline $3 \mathrm{c}$ & $166.9 \pm 29.0$ & $131.2 \pm 33.3$ & $\mathbf{0 . 0 3}$ & $143.2 \pm 51.7$ & $112.0 \pm 13.8$ & 0.53 \\
\hline
\end{tabular}

\section{Effect of insight}

The schizophrenia group consisted of three insight subgroups: poor $(n=10)$, fair $(n=5)$ and $\operatorname{good}(\mathrm{n}=9)$. When the three insight subgroups were included in the analysis, there were significant differences in the Nv of oligodendrocytes in layer 3 of BA 39 but not in the BA 40 compared to the control group. There were no significant differences in the white matter of BA 39/40.

Since there were only 5 cases with fair insight and since this group was also thought to have some impairment of insight, we combined the subgroups with poor and fair insight for statistical analysis. The comparison of the control group with poor+fair and good insight subgroups showed a significant effect of insight on the $\mathrm{Nv}$ of oligodendrocytes in BA 39 in $3 b$ and $3 c$ (Table 4). There were no significant differences in $3 \mathrm{a}$, in each sublayer of BA 40, and in the white matter underlying layer 6 of BA 39 and BA 40. A post hoc test showed a significant decrease of the parameter in the poor+fair subgroup compared to the controls in $3 b(-21 \%, p=0.04)$ and $3 c(-22 \%, p=0.04)$. Moreover, in $3 c$ the $\mathrm{Nv}$ of oligodendrocytes showed a trend to decrease in the poor+fair subgroup compared to the subgroup with good insight $(-20 \%, \mathrm{p}=$ $0.055)$. The subgroup with good insight did not differ from the control group.

\section{Potentially confounding factors}

There were no correlations between age, postmortem interval, refrigerator interval, brain weight, brain $\mathrm{pH}$, lifetime antipsychotics, age at onset or duration of disease and the $\mathrm{Nv}$ of oligodendrocytes in BA 39 or BA 40. 
$\Delta$ controls, $n=24$

O schizophrenia, fair+poor insight, $n=15$

$\square$ schizophrenia, good insight, $\mathrm{n}=\mathbf{9}$

- means
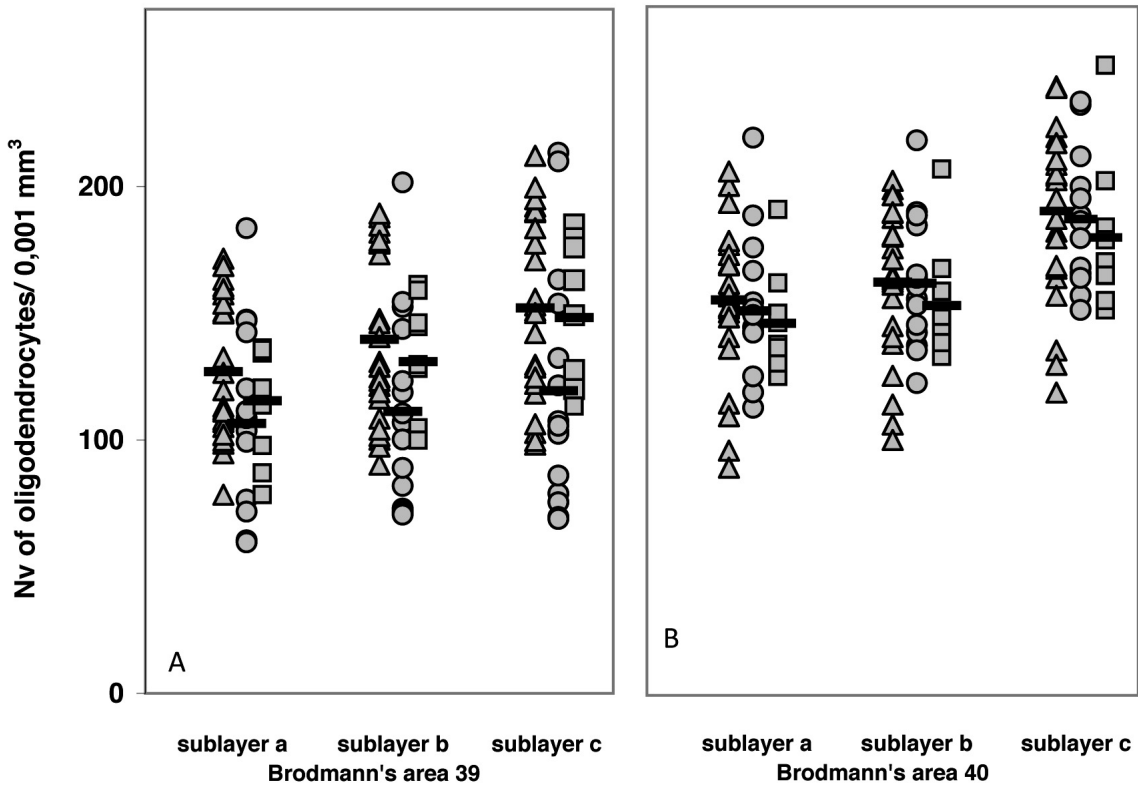

Figure 2. Plots of individual values and means (lines) of the Nv of oligodendrocytes

in the three sublayers of layer 3 in control group and in the insight subgroups. BA 39 (A), BA 40 (B).

Table 4

Effect of insight on the Nv of oligodendrocytes

\begin{tabular}{lccccc} 
Layers & $\begin{array}{c}\text { Controls } \pm \\
\mathrm{SD}(\mathrm{n}=24)\end{array}$ & $\begin{array}{c}\text { Poor+fair insight } \pm \\
\mathrm{SD}(\mathrm{n}=15)\end{array}$ & $\begin{array}{c}\text { Good insight } \pm \\
\mathrm{SD}(\mathrm{n}=9)\end{array}$ & $\mathrm{F}(2.45)$ & $\mathrm{p}$ \\
\hline 3a & Brodmann's area 39 & & $115.36 \pm 22.6$ & 2.13 & 0.13 \\
3b & $126.95 \pm 28.0$ & $106.51 \pm 37.3$ & $130.91 \pm 23.7$ & 3.31 & $\mathbf{0 . 0 4 5}$ \\
3c & $139.66 \pm 33.4$ & $111.20 \pm 38.6$ & $148.20 \pm 28.6$ & 3.54 & $\mathbf{0 . 0 3 7}$ \\
\hline White matter & $412.64 \pm 69.3$ & $119.40 \pm 47.0$ & $407.91 \pm 43.9$ & 1.15 & 0.3 \\
\hline & $152.01 \pm 35.1$ & $383.41 \pm 48.9$ & $145.77 \pm 20.0$ & 0.4 & 0.7 \\
\hline 3a & $154.88 \pm 29.7$ & $150.57 \pm 28.3$ & $152.72 \pm 22.9$ & 0.4 & 0.7 \\
3b & $161.96 \pm 29.0$ & $161.50 \pm 25.0$ & $179.48 \pm 29.6$ & 0.4 & 0.7 \\
\hline c & $189.76 \pm 37.2$ & $186.69 \pm 24.7$ & $393.4 \pm 40.3$ & 0.4 & 0.7 \\
\hline White matter & $386.74 \pm 54.1$ & $401.0 \pm 46.2$ & & & \\
\hline
\end{tabular}




\section{Discussion}

To our knowledge, this is the first study to investigate oligodendrocyte density and effect of insight on oligodendrocyte density in the IPL in subjects with schizophrenia.

\section{Patients versus controls}

We demonstrated a $15 \%$ reduction in the $\mathrm{Nv}$ of oligodendrocytes in each sublayer of layer 3 of BA 39 in the schizophrenia group compared to controls. There were no changes in the $\mathrm{Nv}$ of oligodendrocytes in layer 3 of BA 40 or in the white matter underlying BA 39/40. These findings replicate and extend the results of our previous studies reporting pronounced $\sim 25 \%$ decrease in the oligodendrocyte density in layer 6 of BA 9 using the Stanley Foundation Neuropathology Consortium collection ${ }^{29}$ and in layer 6 and in the white matter of BA 10 using our brain collection $^{30}$. The deficit of oligodendrocytes in BA 9 was associated with a loss of perineuronal oligodendrocytes in $3 \mathrm{a}, 3 \mathrm{~b}$ and $3 \mathrm{c}^{25}$. Our data are also consistent with the prominent reduction of oligodendroglial cells (using Nissl staining and CNPase immunocytochemistry) in layer 3 of BA 9 previously reported in schizophrenia ${ }^{22}$.

We did not find significant differences in the Nv of oligodendrocytes in the white matter in BA 39/40 in schizophrenia. The results of imaging studies of the white matter in the IPL are controversial. Zhou et al. ${ }^{4}$ reported no changes in the white matter volume of the parietal regions in schizophrenia subjects. However, a reduced white matter integrity in the IPL ${ }^{20}$ and a significantly greater decrease over time in frontal gray and parietal white matter ${ }^{6}$ have been reported in schizophrenia patients. These data together with the present data suggest that the PFC might be more se- verely affected than IPL with respect to oligodendrocyte density in schizophrenia.

The reduction in oligodendrocyte density in IPL BA 39 and in the PFC in schizophrenia is consistent with MRI and PET studies that have demonstrated smaller gray matter volumes $^{31}$. These data suggest that the deficit of oligodendrocytes in the gray matter of both cortical areas might contribute to the dysfunction of these cortical areas in schizophrenia.

\section{Oligodendrocyte density and insight}

As hypothesized, we found a significant reduction of oligodendrocyte density in BA 39 in sublayers $3 b$ and $3 c$ in the poor+fair insight subgroup compared to the control group. The differences between subgroup with poor/fair insight and with good insight approached statistical significance $(\mathrm{p}=0.055)$, and subjects with good insight did not differ from controls. Our results are in agreement with the theory that unawareness of illness in schizophrenia may be due to atrophy of the IPL $^{18}$ and of the PFC ${ }^{7,18}$. The data suggest that lack of insight in schizophrenia may be associated with a deficit in oligodendrocytes in the grey matter of the IPL.

The IPL is implicated in neurocognitive impairment in schizophrenia ${ }^{32}$. Recent studies suggest that oligodendrocyte deficits and myelin dysfunctions might contribute to abnormal neuronal connectivity that could in turn lead to cognitive impairment in schizophrenia $^{33}$. Poor insight in schizophrenia is associated with cognitive dysfunction and more severe negative symptoms ${ }^{18,27,34}$. Such data are consistent with the results of our studies of the PFC BA 10 that detected a deficit of pericapillary oligodendrocytes in the subgroup of schizophrenia subjects with predominantly negative symptoms but not in 
the subjects with predominantly positive symptoms compared to controls ${ }^{26}$. They are also consistent with a significant increase in the volume fraction of heterochromatin in the oligodendrocyte nucleus seen only in cases with predominantly negative symptoms relative to controls ${ }^{24}$. Peters and Sethares ${ }^{35}$ reported that in the PFC of monkeys, age-related alterations of myelinated fibers significantly correlated with the cognitive impairment index. Taken together with the results of the present study, these data suggest that the deficit of oligodendrocytes might contribute to cognitive disturbances and impaired insight in schizophrenia.

\section{Oligodendrocyte density and asymmetry}

We found that the oligodendrocyte density in BA 39 showed an asymmetry $(\mathrm{L}>\mathrm{R})$ in the control group but not in schizophrenia group. This result is in agreement with the neuroimaging data of Frederikse et al..$^{36}$ that the volume of the IPL in males is normally asymmetric $(\mathrm{L}>\mathrm{R})$, and this asymmetry is decreased in schizophrenia ${ }^{3,36}$. A functional MRI study ${ }^{37}$ also demonstrated that the largest functional asymmetries $(\mathrm{L}>\mathrm{R})$ are in the IPL in healthy controls but not in schizophrenia patients during the resting state. We did not find any asymmetry in the oligodendrocyte density in BA 40 or in the white matter underlying BA 39/40. Our results provide evidence for the first time that oligodendrocyte density contributes to the normal asymmetry in the angular gyrus.

\section{Effects of confounding factors}

We did not find any effects of age, postmortem delay, $\mathrm{pH}$, brain weight, age at disease onset, or duration of disease on the $\mathrm{Nv}$ of oligodendrocytes. No significant correlation between oligodendrocyte density and total neuroleptic usage was found. This result is in agreement with some experimental data. Konopaske et al. ${ }^{38}$ found a nonsignificant lower oligodendrocyte number in parietal grey matter after chronic exposure of monkeys to haloperidol or olanzapine. However, protective effects of neuroleptics on oligodendrocytes and stimulation of oligodendrocyte proliferation by neuroleptics have recently been reported $^{39}$. Together these data suggest that the reduction in oligodendrocyte density found in the present study is not attributable to neuroleptic exposure.

\section{Limitations and future studies}

There are several limitations to this study. First, this is a preliminary study and should be replicated with more subjects with insight ratings. Females should also be included. Second, it was difficult to estimate the effects of lifetime alcohol and drug abuse in this study: there were six alcohol and drug abuse subgroups based on qualitative criteria (from none to heavy/present), and the number of subjects per each subgroup was not comparable in the control and schizophrenia groups. Third, we found nonsignificant changes in BA 40. A possible reason is that the cytoarchitectonic areas of the human IPL are highly variable. The IPL consists of seven cytoarchitectonically distinct areas: five in the BA 40, and two in BA $39^{40}$.

In summary, we present evidence for a reduction of the oligodendrocyte density in IPL BA 39 in schizophrenia and in subjects having poor+fair insight compared to the control group. These findings suggest that the oligodendroglial deficit in the gray matter of the IPL might be associated with impaired insight in schizophrenia. 
Future studies of the neurobiological cellular basis of poor insight and the role of oligodendrocytes in impaired insight and other cognitive disturbances in schizophrenia will be useful to develop new therapeutic strategies for patients with schizophrenia.

\section{Acknowledgments}

The authors would like to thank the Stanley Medical Research Institute for support this work. Postmortem brain sections were donated by Dr. M.J Webster from the Stanley "Parietal Collection". We express our gratitude to Dr. E. Fuller Torrey for examination of clinical records and editing the manuscript and Dr. M.J. Webster for editing the manuscript.

\section{References}

1. Kaplan RD, Szechtman H, Franco S, Szechtman B, Nahmias C, Garnett ES, et al. Three clinical syndromes of schizophrenia in untreated subjects: relation to brain glucose activity measured by positron emission tomography (PET). Schizophr Res 1993; 11:47-54.

2. Petrides M, Pandya DN. Projections to the frontal cortex from the posterior parietal region in the rhesus monkey. J Comp Neurol 1984; 228: 105-116.

3. Torrey EF. Schizophrenia and the inferior parietal lobule. Schizophr Res 2007; 97: 215-225.

4. Zhou SY, Suzuki M, Takahashi T, Hagino H, Kawasaki Y, Matsui M, et al. Parietal lobe volume deficits in schizophrenia spectrum disorders. Schizophr Res 2007; 89: 35-48.

5. Antonius D, Prudent V, Rebani Y, D'Angelo D, Ardekani BA, Malaspina D, et al. White matter integrity and lack of insight in schizophrenia and schizoaffective disorder. Schizophr Res 2011; 128: 76-82.

6. Olabi B, Ellison-Wright I, McIntosh AM, Wood SJ, Bullmore E, Lawrie SM. Are there progressive brain changes in schizophrenia? A meta-analysis of structural magnetic resonance imaging studies. Biol Psychiatr 2011; 70: 88-96.
7. Larøi F, Fannemel M, Rønneberg U, Flekkøy K, Opjordsmoen S, Dullerud R, et al. Unawareness of illness in chronic schizophrenia and its relationship to structural brain measures and neuropsychological tests. Psychiatr Res 2000; 100: 49-58.

8. Takai A, Uematsu M, Ueki H, Sone K, Kaiya H. Insight and its related factors in chronic schizophrenic patiens: a preliminary study. Eur J Psychiatr 1992; 6: 159-170.

9. Flashman LA, McAllister TW, Andreasen NC, Saykin AJ. Smaller brain size associated with unawareness of illness in patients with schizophrenia. Am J Psychiatry 2000; 157: 1167-1169.

10. Buchy L, Ad-Dab'bagh Y, Malla A, Lepage C, Bodnar M, Joober R, et al. Cortical thickness is associated with poor insight in first-episode psychosis. J Psychiatr Res 2011; 45: 781-787.

11. Flashman LA, McAllister TW, Johnson SC, Rick JH, Green RL, Saykin AJ. Specific frontal lobe subregions correlated with unawareness of illness in schizophrenia: a preliminary study. J Neuropsychiatry Clin Neurosci 2001; 13: 255-257.

12. Shad MU, Muddasani S, Prasad K, Sweeney JA, Keshavan MS. Insight and prefrontal cortex in first-episode Schizophrenia. Neuroimage 2004; 22: 1315-1320.

13. Shad MU, Tamminga CA, Cullum M, Haas GL, Keshavan MS. Insight and frontal cortical function in schizophrenia: a review. Schizophr Res 2006; 86: 54-70.

14. Sapara A, Cooke M, Fannon D, Francis A, Buchanan RW, Anilkumar AP, et al. Prefrontal cortex and insight in schizophrenia: a volumetric MRI study. Schizophr Res 2007; 89: 22-34.

15. Ha TH, Youn T, Ha KS, Rho KS, Lee JM, Kim IY, et al. Gray matter abnormalities in paranoid schizophrenia and their clinical correlations. Psychiatry Res 2004; 132: 251-260.

16. Morgan KD, Dazzan P, Morgan C, Lappin J, Hutchinson G, Suckling J, et al. Insight, grey matter and cognitive function in first-onset psychosis. Br J Psychiatry 2010; 197: 141-148.

17. Raij TT, Riekki TJ, Hari R. Association of poor insight in schizophrenia with structure and function of cortical midline structures and frontopolar cortex. Schizophr Res 2012; 139: 27-32.

18. Cooke MA, Fannon D, Kuipers E, Peters E, Williams SC, Kumari V. Neurological basis of poor insight in psychosis: a voxel-based MRI study. Schizophr Res 2008; 103 : 40-51.

19. Lee KH, Brown WH, Egleston PN, Green RD, Farrow TF, Hunter MD, et al. A functional magnetic resonance 
imaging study of social cognition in schizophrenia during an acute episode and after recovery. Am J Psychiatry 2006; 163 : 1926-1933.

20. Antonius D, Prudent V, Rebani Y, D'Angelo D, Ardekani BA, Malaspina D, et al. White matter integrity and lack of insight in schizophrenia and schizoaffective disorder. Schizophr Res 2011; 128: 76-82.

21. Faget-Agius C, Boyer L, Padovani R, Richieri R, Mundler O, Lançon C, et al. Schizophrenia with preserved insight is associated with increased perfusion of the precuneus. J Psychiatry Neurosci. 2012; 37: 297-304.

22. Hof PR, Haroutunian V, Friedrich VL, Byne W, Buitron C, Perl DP, et al. Loss and altered spatial distribution of oligodendrocytes in the superior frontal gyrus in schizophrenia. Biol Psychiatr 2003; 53: 1075-1085.

23. Tkachev D, Mimmack ML, Ryan MM, Wayland M, Freeman T, Jones PB, et al. Oligodendrocyte dysfunction in schizophrenia and bipolar disorder. Lancet 2003; 362: 798805 .

24. Uranova N, Orlovskaya D, Vikhreva O, Zimina I, Kolomeets N, Vostrikov V, et al. Electron microscopy of oligodendroglia in severe mental illness. Brain Res Bull 2001; 55: 597-610.

25. Vostrikov VM, Uranova NA, Orlovskaya DD. Deficit of perineuronal oligodendrocytes in the prefrontal cortex in schizophrenia and mood disorders. Schizophr Res 2007; 94: 273-280.

26. Vostrikov V, Orlovskaya D, Uranova N. Deficit of pericapillary oligodendrocytes in the prefrontal cortex in schizophrenia. World J Biol Psychiatr 2008; 9: 34-42.

27. Parellada M, Boada L, Fraguas D, Reig S, CastroFornieles J, Moreno D, et al. Trait and state attributes of insight in first episodes of early-onset schizophrenia and other psychoses: a 2-year longitudinal study. Schizophr Bull 2011; 37: 38-51.

28. Zilles K. Architecture of the human cerebral cortex In: Paxinos G, Mai JK, eds. The human nervous system. 2nd edition. Elsevier Academic Press; 2004. p. 997-1055.

29. Uranova NA, Vostrikov VM, Orlovskaya DD, Rachmanova VI. Oligodendroglial density in the prefrontal cortex in schizophrenia and mood disorders: a study from the Stanley Neuropathology Consortium. Schizophr Res 2004; 67: 269-275.

30. Vostrikov VM, Uranova NA, Rakhmanova VI, Orlovskaia DD. Lowered oligodendroglial cell density in the prefrontal cortex in schizophrenia. Zh Nevrol Psikhiatr Im SS Korsakova 2004; 104: 47-51 [in Russian].
31. Kubicki M, Shenton ME, Salisbury DF, Hirayasu Y, Kasai K, Kikinis R, et al. Voxel-based morphometric analysis of gray matter in first episode schizophrenia. Neuroimage 2002; 17: 1711-1719.

32. Cleghorn JM, Kaplan RD, Nahmias C, Garnett ES, Szechtman H, Szechtman B. Inferior parietal region implicated in neurocognitive impairment in schizophrenia. Arch Gen Psychiatr 1989; 46: 758-760.

33. Konrad A, Winterer G. Disturbed structural connectivity in schizophrenia primary factor in pathology or epiphenomenon? Schizophr Bull 2008; 34: 72-92.

34. Segarra Echebarria R, Ojeda del Pozo N, Zabala Rabadan A, García Ormaza J, Peña Lasa J, Eguíluz Uruchurtu I, et al. Insight in first episode psychosis. Conceptual and clinical considerations. Eur J Psychiat 2010; 24: 78-86.

35. Peters A, Sethares C. Aging and the myelinated fibers in prefrontal cortex and corpus callosum of the monkey. $\mathrm{J}$ Comp Neurol 2002; 442: 277-291.

36. Frederikse ME, Lu A, Aylward E, Barta P, Pearlson G.. Sex differences in the inferior parietal lobule. Cereb Cortex 1999; 9: 896-901.

37. Swanson N, Eichele T, Pearlson G, Kiehl K, Yu Q, Calhoun VD. Lateral differences in the default mode network in healthy controls and patients with schizophrenia. Hum Brain Mapp 2011; 32: 654-664.

38. Konopaske GT, Dorph-Petersen KA, Sweet RA, Pierri JN, Zhang W, Sampson AR, et al. Effect of chronic antipsychotic exposure on astrocyte and oligodendrocyte numbers in macaque monkeys. Biol Psychiatr 2008; 63: 759-765.

39. Wang H, Xu H, Niu J, Mei F, Li X, Kong J, et al. Haloperidol activates quiescent oligodendroglia precursor cells in the adult mouse brain. Schizophr Res 2010; 119: 164-174.

40. Caspers S, Eickhoff SB, Geyer S, Scheperjans F, Mohlberg H, Zilles K, et al. The human inferior parietal lobule in stereotaxic space. Brain Struct Funct 2008; 212: 481495.

Corresponding author:

Victor Vostrikov

Laboratory of Clinical Neuropathology

Mental Health Research Center

Zagorodnoe shosse 2, 117152, Moscow, Russia

Tel.: +7(495)9528730

Fax: +7(495)9528940

E-mail: vostrikovvm@mail.ru 